\title{
Effects of cigarette smoking on blood lipids in Korean men: Cardiovascular and Metabolic Diseases Etiology Research Center cohort
}

\author{
Soo Kyoung Kim ${ }^{1}$, Hyeon Chang $\mathrm{Kim}^{1,2,3}$, Jee-Seon Shim²,3, and Dae Jung Kim ${ }^{4,5}$
}

\begin{abstract}
${ }^{1}$ Department of Public Health, Yonsei University Graduate School, Seoul; ${ }^{2}$ Department of Preventive Medicine, ${ }^{3}$ Cardiovascular and Metabolic Diseases Etiology Research Center, Yonsei University College of Medicine, Seoul; ${ }^{4}$ Department of Endocrinology and Metabolism, ${ }^{5}$ Cardiovascular and Metabolic Diseases Etiology Research Center, Ajou University School of Medicine, Suwon, Korea
\end{abstract}

Received: April 19, 2019

Revised : July 16, 2019

Accepted: August 27, 2019

\section{Correspondence to}

Hyeon Chang Kim, M.D.

Department of Preventive Medicine, Yonsei University

College of Medicine, 50-1

Yonsei-ro, Seodaemun-gu, Seoul

03722, Korea

Tel: +82-2-2228-1873

Fax: +82-2-392-8133

E-mail: hckim@yuhs.ac

https:/orcid.org/oooo-ooo1-

7867-1240
Background/Aims: Cigarette smoking and abnormal blood lipids are major risk factors for cardiovascular disease. The results of previous studies on the relationship between cigarette smoking and dyslipidemia are controversial. In the present study, we investigated the independent association between cigarette smoking and blood lipid levels in a male Korean population.

Methods: A total of 1,932 men aged from 30 to 64 years old participated in the Cardiovascular and Metabolic Diseases Etiology Research Center cohort study. Smoking history was obtained by in-person interviews. In all regression models, measurements of triglyceride levels were log-transformed.

Results: Triglyceride levels were higher in current smokers than in never-smokers (median: $149 \mathrm{mg} / \mathrm{dL}$ vs. $115 \mathrm{mg} / \mathrm{dL}, p<0.001$ ) even after adjusting age, body mass index, alcohol intake, systolic blood pressure, fasting glucose, physical activity, and nutrition intake $(\beta=0.14, p<0.001)$. We further divided people into heavy and light smokers using 20 pack-years as the cut-off. Higher triglyceride were found in current heavy smokers $(\beta=0.18, p<0.001)$, current light smokers $(\beta=0.13, p<0.001)$, as well as in past heavy smokers $(\beta=0.08, p=0.037)$, as compared to never-smokers. Moreover, significantly lower high-density lipoprotein cholesterol (HDL-C) were observed in current heavy smokers $(\beta=-2.27 \mathrm{mg} / \mathrm{dL}, p=0.009)$.

Conclusions: Cigarette smoking is associated with higher triglyceride in Korean men, with the most dramatic effect seen in current smokers with a smoking history of more than 20 pack-years. HDL-C were also lower in current smokers with more than 20 pack-years.

Keywords: Cigarette smoking; Dyslipidemia; Triglyceride; Lipoproteins

\section{INTRODUCTION}

Dyslipidemia is represented by high blood levels of total cholesterol ( $\mathrm{TC} \geq 240 \mathrm{mg} / \mathrm{dL}$ ), low-density lipoprotein cholesterol (LDL-C $\geq 160 \mathrm{mg} / \mathrm{dL}$ ), and triglyceride ( $\mathrm{TG} \geq$ $200 \mathrm{mg} / \mathrm{dL}$ ) and a low level of high-density lipoprotein cholesterol (HDL-C < $40 \mathrm{mg} / \mathrm{dL}$ ) [1]. The serum lipid components are associated with cardiovascular disease (CVD) risk both independently and in combination [2-4]. Cigarette smoking is also a well-known major risk factor for atherosclerotic change and CVD $[2,5,6]$. The results of previous studies suggest that cigarette smoking can produce oxygen-derived free radicals that damage blood lipids. These damaged lipids may promote atherogenesis and lead to atherosclerosis [7]. However, the results vary in different studies, and the effect of smoking on 
lipid levels is not well defined [8-10].

The prevalence of dyslipidemia among Korean population has been increasing for last 5 years. Nearly four out of $10(40.5 \%)$ adults aged over 30 years old are reported to have dyslipidemia, and the degree is much higher in men; overall, nearly half (47.9\%) of Korean men aged over 30 years old are reported have dyslipidemia [11]. According to Organization for Economic Cooperation and Development Health Statistics 2017, the daily smoking rate among Korean men is $31 \%$, which is still higher compared to that of average, $23 \%$ [12]. The present study aims to examine the independent relationship between cigarette smoking and blood lipid levels within a male Korean population.

\section{METHODS}

\section{Study population}

Participants from the Cardiovascular and Metabolic Diseases Etiology Research Center (CMERC) cohort provided the sample population for this study [13]. The participants were aged between 30 and 64 years old and were free from cancer, myocardial infarction, cerebral stroke, and heart failure. Health examination of the participants was conducted between 2013 and 2018 at medical centers in Seoul and Suwon. The study inclusion flow chart is shown in Fig. 1. Of the total of 8,097 CMERC participants, 2,808 were men. Women were excluded from the analysis because the smoking rate among women was too low to reveal the association between smoking and serum lipid levels $(2.88 \%$ were current smokers and $3.20 \%$ were past smokers). Of the men, 580 reported that they were taking drugs for the treatment of hyperlipidemia, statin for the management of lipid levels, or omega-3 fatty acids supplementations. These patients were excluded from the sample. A further 77 past smokers were excluded because their smoking cessation periods were less than 1 year. Finally, 219 men were excluded because they had missing values for potential confounding variables. After these exclusions, a total of 1,932 men were included in our study population (Fig. 1).

\section{Measurements}

Trained interviewers obtained socio-demographic in-

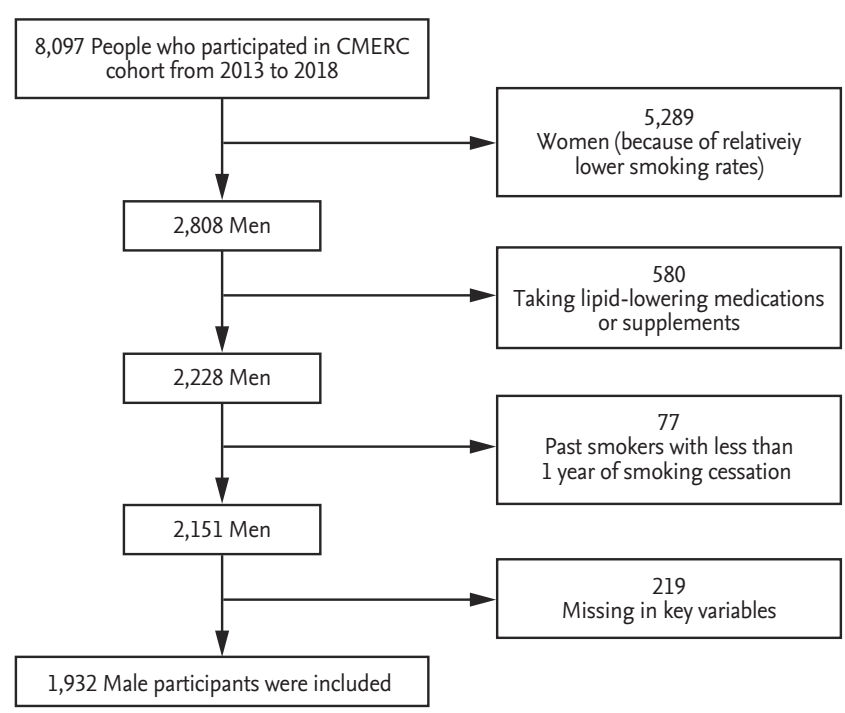

Figure 1. Study inclusion flow chart. CMERC, Cardiovascular and Metabolic Diseases Etiology Research Center.

formation, medical, and lifestyle history in in-person interviews. Never-smokers were defined as people who reported they had smoked no more than 100 cigarettes in their lifetime. Past smokers were defined as people who reported having smoked $\geq 100$ cigarettes, but did not smoke at the time of the present investigation. However, as mentioned above, past smokers with less than 1 year of smoking cessation period were excluded from the analysis. This was done because, as demonstrated by previous studies, it is at least after a year after smoking cessation that the characteristics such as the risk of CVD incidence [14] or the likelihood of relapse [15] of past smokers become distinctively different from those of current smokers. Therefore, we assumed 'past smokers' to be individuals whose smoking cessation period had started more than a year ago. Current smokers were defined as those who had smoked $\geq 100$ cigarettes and who smoked at the time of our investigation. Pack-years were calculated for each participant by multiplying packs smoked per day by years as a smoker. For example, 1 pack-year means that the participant smoked one pack of cigarettes every day for a year. While there is no general consensus on the definition of heavy smoking, we used pack-year of 20 as the cut-off in the present study. The same criterion was used in previous studies on the relationship between smoking and the risk of cancer or CVD [16-18]; in addition, in a previous study, the stan- 
dard of 20 pack-year was deemed to be an appropriate measure to define heavy smoking in terms of nicotine dependency or difficulty in smoking cessation [19].

The participants were also asked "Are you currently drinking alcohol?" If they said "No," they were considered as non-drinkers. Others were considered as current drinkers. Among current drinkers, people who said they drank more than twice a week and also drank more than 7 units of alcohol at once were considered to be highrisk drinkers.

Information about dietary intake was obtained by using a semi-quantitative food frequency questionnaire developed for the Korea National Health and Nutrition Examination Survey [2o]. Daily total energy intake (kcal), carbohydrate intake (g), and fat intake (g) were calculated. The participants were asked about the average consumption frequency and the amounts of 112 types of food consumed throughout the preceding year. Physical activity was measured by the Korean version of the International Physical Activity Questionnaire (IPAQ)-Short Form. The participants were divided into three groups according to IPAQ guidelines: low, moderate, and high [21,22].

The participants were asked to fast for a minimum of 8 hours before visiting health examination center. Then, the participants' blood samples were collected, and laboratory tests were performed in a central laboratory (Seoul Clinical Laboratories R\&D Center, Seoul, Korea). Serum fasting glucose was measured using a colorimetric method and an autoanalyzer (ADVIA 1800 Auto Analyzer, Siemens Medical Solutions, Malvern, PA, USA). Serum TC, HDL-C, and TG were measured using automated enzymatic techniques. LDL-C was calculated using Friedewald's equation [23].

Body weight and height were measured using standardized techniques and equipment. The participants were asked to wear light indoor clothing without shoes for weight and height measurements. Body mass index (BMI) was calculated as weight divided by height squared $\left(\mathrm{kg} / \mathrm{m}^{2}\right)$. Blood pressure was measured three times after a 5-minute rest in the sitting position via the right arm using an electronic manometer (HEM-708oIC, Omron Healthcare Co. Ltd., Kyoto, Japan). The average of the three values was used for further analysis.

\section{Ethics statement}

All participants provided written informed consent, and the study protocol was approved by the Institutional Review Boards of Severance Hospital, Yonsei University Health System, Seoul, Korea (4-2013-0661) and Ajou University Hospital, Suwon, Korea (AJIRB-BMRSUR-13-272).

\section{Statistical analyses}

Basic characteristics of the study population are presented as means with standard deviation, medians with interquartile range, or numbers with percentage. T-test and chi-square test were used to identify significant relationships between basic characteristics and smoking status. Multiple linear regression analysis was used to examine the association between cigarette smoking and each serum lipid level. In all regression analyses, the never-smoker group was used as the reference group. Due to their right-skewed distributions, serum glucose and TG levels were log-transformed to satisfy the normality assumption for statistical analysis. For HDL-C and TG levels, the least squares means, estimated from linear regression models, were derived for convenience of interpretation. Least squares mean for one variable is the adjusted mean value that controls the effect of the major covariates [24]; therefore, we considered it suitable for comparing the mean lipid levels of each smoker group after controlling the influence of the covariates. Age, BMI, alcohol intake, systolic blood pressure, fasting serum glucose, physical activity, and dietary (total energy, carbohydrates, and fat) intake were adjusted as potential covariates in the regression analyses and least squares means analyses. To determine whether individuals with a higher smoking amount, as measured by pack-years, have higher blood lipid levels than those without such amount, we conducted linear trend tests and multiple comparisons using Tukey's method. All statistical analyses were performed using SAS version 9.4 (SAS Institute, Cary, NC, USA) and $p<0.05$ was considered to be statistically significant.

\section{RESULTS}

\section{General characteristics}

Table 1 shows the general characteristics of the study population. The mean age was 49.5. Past smokers and current smokers amounted to $40.3 \%(\mathrm{n}=779)$ and $36.5 \%$ 
Table 1. General Characteristics of study population

\begin{tabular}{|c|c|c|c|c|c|c|}
\hline \multirow[b]{2}{*}{ Characteristic } & \multirow{2}{*}{$\begin{array}{c}\text { Total } \\
(\mathrm{n}=1,932)\end{array}$} & \multicolumn{5}{|c|}{ Smoking status } \\
\hline & & $\begin{array}{c}\text { Never-smoker } \\
(\mathrm{n}=448)\end{array}$ & $\begin{array}{l}\text { Past smoker } \\
(\mathrm{n}=779)\end{array}$ & $p$ value ${ }^{a}$ & $\begin{array}{l}\text { Current smoker } \\
(\mathrm{n}=705)\end{array}$ & $p$ value $^{\mathrm{a}}$ \\
\hline Age, yr & $49 \cdot 5 \pm 9.6$ & $48.4 \pm 10.5$ & $52.8 \pm 8.3$ & $<0.001$ & $46.5 \pm 9.2$ & 0.002 \\
\hline Weight, kg & $72.8 \pm 10.2$ & $72.0 \pm 9.5$ & $72.0 \pm 9.8$ & 0.977 & $74.1 \pm 10.9$ & 0.001 \\
\hline Height, cm & $170.9 \pm 6.0$ & $170.7 \pm 6.2$ & $170.0 \pm 5.9$ & 0.060 & $171.9 \pm 5.9$ & 0.001 \\
\hline Body mass index, $\mathrm{kg} / \mathrm{m}^{2}$ & $24.9 \pm 2.9$ & $24.7 \pm 2.8$ & $24.9 \pm 2.7$ & 0.277 & $25.0 \pm 3.1$ & 0.055 \\
\hline Systolic blood pressure, mmHg & $124 \cdot 4 \pm 13 \cdot 7$ & $123.4 \pm 13.1$ & $125.8 \pm 14.0$ & 0.003 & $123.6 \pm 13.6$ & 0.862 \\
\hline Diastolic blood pressure, $\mathrm{mmHg}$ & $80.3 \pm 10.0$ & $79 \cdot 5 \pm 9.5$ & $81.0 \pm 10.1$ & 0.012 & $80.0 \pm 10.1$ & 0.449 \\
\hline Fasting serum glucose, mg/dL & $94(87-103)$ & $91(85-100)$ & $96(89-105)$ & $<0.001$ & $94(86-102)$ & 0.006 \\
\hline $\mathrm{TC}, \mathrm{mg} / \mathrm{dL}$ & $196.5 \pm 33.6$ & $194.9 \pm 33.6$ & $196.6 \pm 34.0$ & 0.375 & $197 \cdot 4 \pm 33.0$ & 0.213 \\
\hline HDL-C, mg/dL & $50.2 \pm 12.2$ & $50.6 \pm 12.5$ & $50.8 \pm 12.4$ & 0.798 & $49 \cdot 3 \pm 11.79$ & 0.077 \\
\hline LDL-C, mg/dL & $114.7 \pm 31.3$ & $116.4 \pm 30.9$ & $115 \cdot 4 \pm 31.1$ & 0.614 & $112.8 \pm 31.7$ & 0.059 \\
\hline $\mathrm{TG}, \mathrm{mg} / \mathrm{dL}$ & $133(93-188)$ & $115(84-168)$ & $130(92-176)$ & 0.027 & $149(103-210)$ & $<0.001$ \\
\hline Energy intake, kcal/day & $2,670.5 \pm 925.7$ & $2,504.9 \pm 856.7$ & $2,544 \cdot 5 \pm 817 \cdot 4$ & 0.422 & $2,915 \cdot 1 \pm 1,025 \cdot 3$ & $<0.001$ \\
\hline Carbohydrate intake, g/day & $417.2 \pm 128.5$ & $403.9 \pm 119.5$ & $405.6 \pm 119.7$ & 0.816 & $438.4 \pm 140.3$ & $<0.001$ \\
\hline Protein intake, g/day & $83.5 \pm 37.2$ & $80.6 \pm 35.7$ & $80.1 \pm 32.8$ & 0.787 & $89.2 \pm 41.7$ & $<0.001$ \\
\hline Fat intake, g/day & $50.4 \pm 28.5$ & $48.4 \pm 27.8$ & $46.8 \pm 24.6$ & 0.304 & $55 \cdot 7 \pm 31.9$ & $<0.001$ \\
\hline \multicolumn{7}{|l|}{ Current alcohol intake } \\
\hline Non-drinker & $97(5 \cdot 0)$ & $29(6.5)$ & $58(7 \cdot 5)$ & $<0.001$ & $10(1.4)$ & $<0.001$ \\
\hline Moderate drinker & $1,175(60.8)$ & $332(74.1)$ & $488(62.6)$ & & $355(50.4)$ & \\
\hline High-risk drinker & $660(34.2)$ & $87(19 \cdot 4)$ & $233(29.9)$ & & $340(48.2)$ & \\
\hline \multicolumn{7}{|l|}{ Physical activity } \\
\hline Low & $773(40.0)$ & $157(35 \cdot 0)$ & $292(37.5)$ & 0.425 & $324(46.0)$ & 0.001 \\
\hline Moderate & $720(37 \cdot 3)$ & $186(41.5)$ & $294(37.7)$ & & $240(34.0)$ & \\
\hline High & $439(22.7)$ & $105(23.4)$ & $193(24.8)$ & & $141(20.0)$ & \\
\hline
\end{tabular}

Values are presented as mean $\pm \mathrm{SD}$, median (interquartile range), or number (\%).

TC, total cholesterol; HDL-C, high-density lipoprotein cholesterol; LDL-C, low-density lipoprotein cholesterol; TG, triglyceride.

${ }^{a} \mathrm{~A} p$ values are calculated by independent $t$ tests or chi-square tests, compared to non-smokers.

( $n=705)$ of the total $(n=1,932)$, respectively. Independent $t$ test and chi-square test were performed to establish whether the general characteristics of past smokers and current smokers differed from those of never-smokers. The results showed that, as compared to never-smokers, past smokers were older, while current smokers were younger, and these differences in age were statistically significant $(p<0.001$ and $p=0.002$, respectively). Systolic and diastolic blood pressure, as well as fasting glucose levels, were significantly higher in past smokers than in never-smokers. Furthermore, current smokers had significantly higher measurements of weight and height, and a higher fasting glucose level than the never-smoker group. For alcohol drinking, current smokers drank more than never-smokers. The proportion of high-risk drinkers was the highest in the current smoker group (48.2\%), which is nearly half, followed by past smoker group (29.9\%) and never-smoker group (19.4\%). In addition, current smokers were less physically active and consumed more calories, carbohydrate, protein, and fat than never-smokers. For blood lipid levels, the TG levels of current and past smokers were significantly much higher as compared to that of never-smokers $34 \mathrm{mg} / \mathrm{dL}$ higher, $p<0.001$ and $15 \mathrm{mg} / \mathrm{dL}$ higher, $p=0.027$, current smokers and past smokers, respectively). For other lipid components, there was no significant difference between each smoker group. 


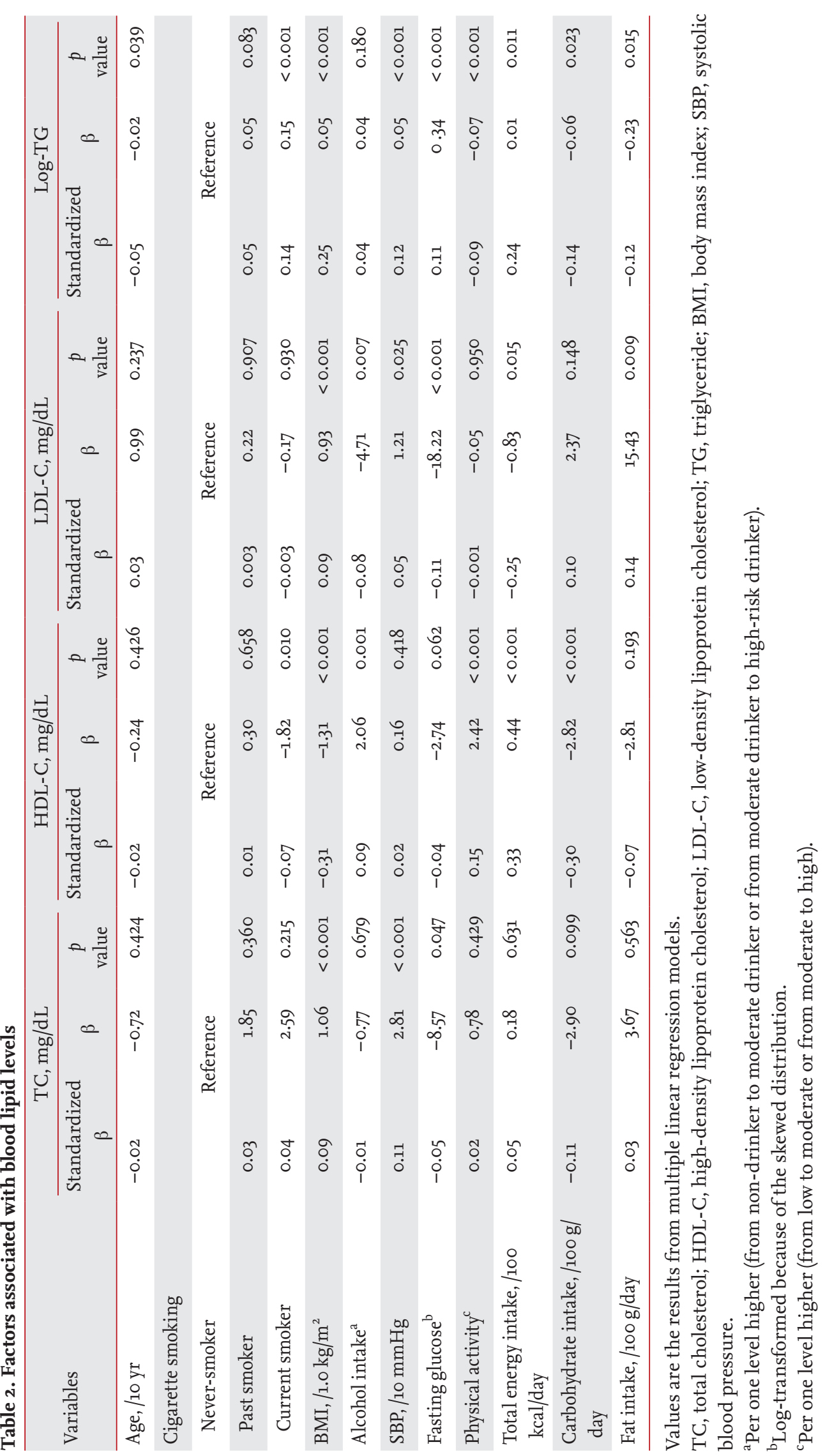




\section{Smoking status and lipid profile}

Associations between blood lipid levels and several variables, including smoking status, were determined using multiple linear regression analysis (Table 2). BMI was significantly strongly associated with altered levels of all serum lipids that were measured. Individuals with higher BMI had higher levels of TC, LDL-C, and logTG, but lower HDL-C levels. Alcohol drinking was positively associated with HDL-C levels, but negatively with LDL-C levels. Systolic blood pressure was positively associated with TC, LDL-C, and log-TG levels. Serum glucose levels were negatively associated with LDL-C levels, but positively associated with log-TG levels. The participants who were more physically active tended to have higher HDL-C and lower log-TG levels as compared to their less active counterparts. Total energy intake was significantly positively associated with HDL-C and $\log$-TG, whereas the association with LDL-C was significantly negative. Carbohydrate intake was significantly associated with lower HDL-C and log-TG levels, while fat intake was significantly associated with higher LDL-C, but lower log-TG.

With regard to the reference group, lipid measurements of past smokers were not significantly different (TC was $1.85 \mathrm{mg} / \mathrm{dL}$ higher, $p=0.360$; HDL-C was 0.30 $\mathrm{mg} / \mathrm{dL}$ higher, $p=0.658$; LDL-C was $0.22 \mathrm{mg} / \mathrm{dL}$ higher, $p=0.907$; and log-TG was 0.05 higher, $p=0.083$ ). In current smokers, HDL-C was significantly lower (1.82 mg/ dL lower, $p=0.010$ ), while log-TG was significantly higher (o.14 higher, $p<0.001)$ than in never-smokers. For TC (2.59 mg/dL higher, $p=0.215)$ and LDL-C $(0.07 \mathrm{mg} / \mathrm{dL}$ lower, $p=0.930$ ), no significant difference between current smokers and never-smokers was observed. In addition, the independent association between the non-logtransformed TG value and smoking status was found to be consistent with the results of the analysis with log-TG (data not shown in table). In past smokers, TG was not significantly different from that of never-smokers (6.62 $\mathrm{mg} / \mathrm{dL}$ higher, standardized $\beta=0.03, p=0.277)$. Current smokers had significantly higher TG than never-smokers $(22.88 \mathrm{mg} / \mathrm{dL}$ higher, standardized $\beta=0.10, p<0.001)$.

Using the method of least squares (adjusted for the same covariates as the preceding analysis), the mean TG level of current smokers was $169.79 \mathrm{mg} / \mathrm{dL}$, which was significantly higher than that of never-smokers $(22.88$ mg/dL higher than never-smokers, $p<0.001$ ) and past smokers (16.26 mg/dL higher than past smokers, $p=$ 0.004). No significant difference in adjusted means of TG level between never-smokers and past smokers was found; an adjusted mean of TG level of past smokers was $153.53 \mathrm{mg} / \mathrm{dL}$ and it was $6.62 \mathrm{mg} / \mathrm{dL}$ higher than that of never-smokers, $p=0.277$. The adjusted mean HDL level of current smokers was $48.94 \mathrm{mg} / \mathrm{dL}$, which was significantly lower than that of never-smokers $(2.56 \mathrm{mg} / \mathrm{dL}$ lower, $p=0.010$ ) and past smokers $(3.39 \mathrm{mg} / \mathrm{dL}$ lower, $p$ $=0.001)$. Similarly to TG level, there was no significant difference in HDL-C level between never-smokers and past smokers; HDL-C level of past smokers $(51.07 \mathrm{mg} / \mathrm{dL})$ which was $0.30 \mathrm{mg} / \mathrm{dL}$ higher than that of never-smokers $(50.77 \mathrm{mg} / \mathrm{dL}, p=0.658)$.

For further analysis, we subdivided each smoker group based on the amount of smoking using 20 pack-years as the cut-off between light and heavy smokers. The participants were divided into the following five groups: (1) never-smokers, (2) past light smokers, (3) past heavy smokers, (4) current light smokers, and (5) current heavy smokers. Table 3 shows the results from multiple linear regression analysis, using the never-smokers as the reference group. Past smokers with fewer than 20 packyears showed no significant difference in blood lipid levels. However, past smokers with more than 20 packyears had a higher log-TG level as compared to that of never-smokers (log-TG was 0.08 higher, $p=0.037$ ). For current smokers, current light smokers (log-TG was 0.13 higher, $p<0.001$ ) and current heavy smokers (log-TG was 0.18 higher, $p<0.001$ ) had significantly higher logTG levels than never-smokers. We observed a higher log-TG level in current heavy smokers, with more than 20 pack-years, than in current light smokers, with fewer than 20 pack-years. The independent association between the non-log-transformed TG value and smoking status with the amount of smoking was additionally evaluated (data not shown in table). Past light smokers and past heavy smokers were not significantly different in terms of their TG level compared to never-smokers (5.75 $\mathrm{mg} / \mathrm{dL}$ higher, $p=0.378$ in past light smokers and 10.59 $\mathrm{mg} / \mathrm{dL}$ higher, $p=0.192)$. At the same time, current light smokers had a significantly higher TG level than never-smokers ( $15.90 \mathrm{mg} / \mathrm{dL}$ higher, $p=0.027)$, and current heavy smokers had a much higher TG level than never-smokers (31.99 mg/dL higher, $p<0.001$ ).

Current heavy smokers also had significantly lower 


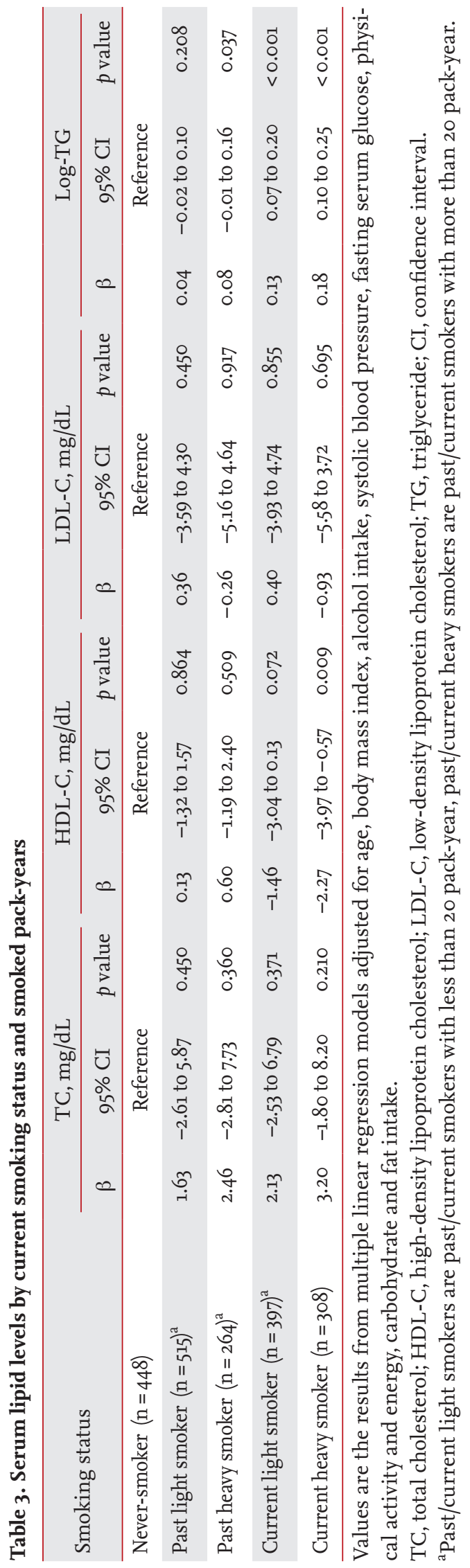

HDL-C levels as compared to those of never-smokers (2.27 mg/dL lower, $p=0.009$ ); however, HDL-C level of current light smokers was not significantly different from that of never-smokers $(1.46 \mathrm{mg} / \mathrm{dL}$ lower than never smokers, $p=0.072$ ).

The least squares means were also calculated for TG levels in each of the five smoker groups that were adjusted for potential variables as described above. The results for this analysis are presented in Table 4. Current heavy smokers had the highest adjusted mean TG levels (least squares means $=178.57 \mathrm{mg} / \mathrm{dL}$ ), and this value was significantly higher than that of current light smokers (mean difference $=16.09 \mathrm{mg} / \mathrm{dL}, p=0.041$ ), past heavy smokers (mean difference $=21.40 \mathrm{mg} / \mathrm{dL}, p=0.013$ ), past light smokers (mean difference $=26.24 \mathrm{mg} / \mathrm{dL}, p<0.001$, and never-smokers (mean difference $=31.99 \mathrm{mg} / \mathrm{dL}, p<$ 0.001). Furthermore, current light smokers had a significantly higher adjusted mean TG levels compared to never-smokers (mean difference $=15.90 \mathrm{mg} / \mathrm{dL}, p=0.027$ ); however, when compared to past smoker groups, the difference did not reach statistical significance. Beyond the significantly higher TG levels in current smokers, there was no significant difference between the adjusted means of the past smoker groups and never- smoker group.

\section{Blood lipid levels for each smoker group according to smoking amounts}

We evaluated whether people with a higher smoking amount have higher serum lipid levels. Due to difficulties in interpretation, past smokers were excluded from this analysis. We first performed linear trend analysis, again dividing current smokers into light and heavy smokers using 20 pack-years as the cut-off. Of the four serum lipids measured, the crude means of HDL-C and TG showed a significant linear trend $(p$ for trend $=$ 0.037 for HDL-C and $p$ for trend < 0.001 for TG). With an increase of the amount of smoking, HDL-C level decreased, while TG level increased. Fig. 2 shows the detailed information on the means of lipid levels of each smoker group.

Next, we performed multiple comparison analysis of the crude means of TG level using Tukey's method and a studentized range of 19.11 (data not shown in table). The results showed a significantly higher TG level in light smokers (mean difference of $31.37 \mathrm{mg} / \mathrm{dL}, p<0.001$ ) 
Table 4. Comparison of blood triglyceride levels between each of the smoking categories

\begin{tabular}{|c|c|c|c|c|}
\hline \multirow{2}{*}{ Name } & \multirow{2}{*}{$\begin{array}{c}\text { Group } 1, \mathrm{mg} / \mathrm{dL} \\
\text { Mean } \pm \mathrm{SE}\end{array}$} & \multirow{2}{*}{$\frac{\text { Group 2, mg/dL }}{\text { Mean } \pm \mathrm{SE}}$} & \multirow{2}{*}{$\begin{array}{c}\text { Group 1-2, mg/dL } \\
\text { Mean }(95 \% \mathrm{CI})\end{array}$} & \multirow{2}{*}{$p$ value } \\
\hline & & & & \\
\hline Current heavy smoker ${ }^{a}$ & $178.57 \pm 5.82$ & & & \\
\hline Current light smoker & & $162.48 \pm 5.30$ & 16.09 (0.70 to 31.48$)$ & 0.041 \\
\hline Past heavy smoker & & $157.17 \pm 6.39$ & $21.40(4.58$ to 38.23$)$ & 0.013 \\
\hline Past light smoker & & $152.33 \pm 4.46$ & 26.24 (11.73 to 40.74$)$ & $<0.001$ \\
\hline Never-smoker & & $146.58 \pm 4.82$ & $31.99(16.90$ to 47.08$)$ & $<0.001$ \\
\hline Current light smoker ${ }^{\mathrm{a}}$ & $162.48 \pm 5.30$ & & & \\
\hline Past heavy smoker & & $157.17 \pm 6.39$ & $5.31(-11.54$ to 22.17$)$ & 0.537 \\
\hline Past light smoker & & $152.33 \pm 4.46$ & $10.15(-3.74$ to 24.04$)$ & 0.152 \\
\hline Never-smoker & & $146.58 \pm 4.82$ & $15.90(1.84$ to 29.96$)$ & 0.027 \\
\hline Past heavy smoker ${ }^{a}$ & $157.17 \pm 6.39$ & & & \\
\hline Past light smoker & & $152.33 \pm 4.46$ & $4.83(-10.27$ to 19.94$)$ & 0.530 \\
\hline Never-smoker & & $146.58 \pm 4.82$ & $10.59(-5.31$ to 26.48$)$ & 0.192 \\
\hline Past light smoker ${ }^{\mathrm{a}}$ & $152.33 \pm 4.46$ & & & \\
\hline Never-smoker & & $146.58 \pm 4.82$ & $5.75(-7.05$ to 18.55$)$ & 0.378 \\
\hline
\end{tabular}

Values are least square means adjusted for age, body mass index, alcohol drinking, systolic blood pressure, log-transformed fasting serum glucose, physical activity and energy, carbohydrate and fat intake.

SE, standard error; CI, confidence interval.

${ }^{\text {a }}$ Past/current light smokers are past/current smokers with less than 20 pack-year, past/current heavy smokers are past/current smokers with more than 20 pack-year.

and heavy smokers (mean difference of $43.99 \mathrm{mg} / \mathrm{dL}, p$ $<0.001$ ), as compared to never-smokers. However, we found no significant difference between the TG levels of light and heavy smokers (mean difference of 12.62 $\mathrm{mg} / \mathrm{dL}, p=0.307$ ). We also conducted multiple comparisons for the (crude) means of HDL-C (data not shown in table). Although HDL-C was low in proportion to the amount of smoked cigarettes, the differences in HDL-C level were not statistically significant (studentized range was 2.08; mean difference between never-smoker and light smoker $=0.85 \mathrm{mg} / \mathrm{dL}, p=0.562$; mean difference between never-smoker and heavy smoker $=1.88 \mathrm{mg} / \mathrm{dL}$, $p=0.090 ;$ mean difference between light smoker and heavy smoker $=1.03 \mathrm{mg} / \mathrm{dL}, p=0.500$ ). We also evaluated the changes of TG and HDL-C levels with an increase of smoked pack-year among current smokers (Fig. 3).

\section{DISCUSSION}

In the present study of apparently healthy Korean men, cigarette smoking was found to be associated with higher

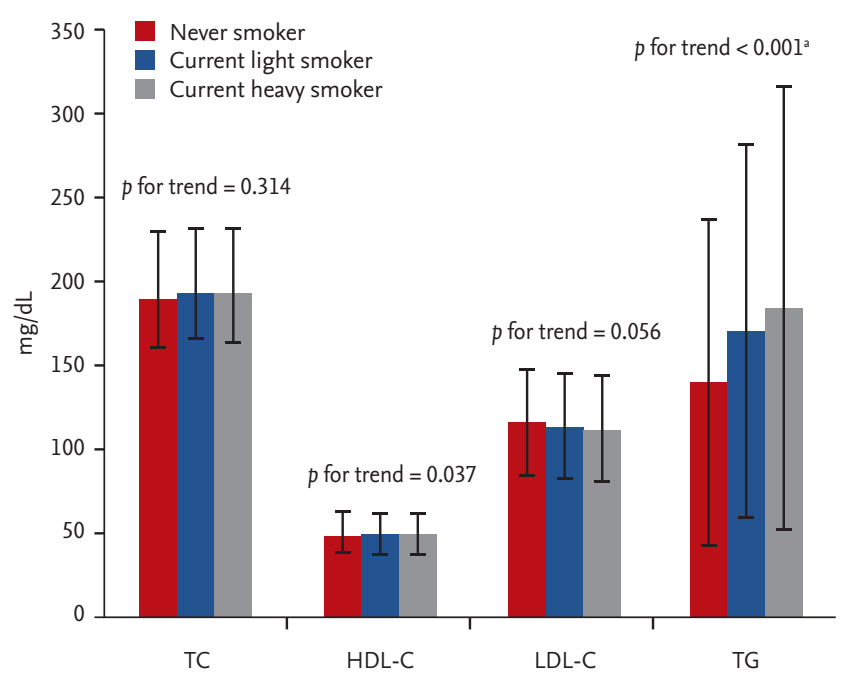

Figure 2. Mean of each lipid level according to current smoking status among Korean men. Past smokers who do not smoke currently were excluded. TC, total cholesterol; HDLC, high-density lipoprotein cholesterol; LDL-C, low-density lipoprotein cholesterol; TG, triglyceride. ${ }^{\mathrm{a}} \mathrm{Cal}-$ culated from log-transformed TG. 

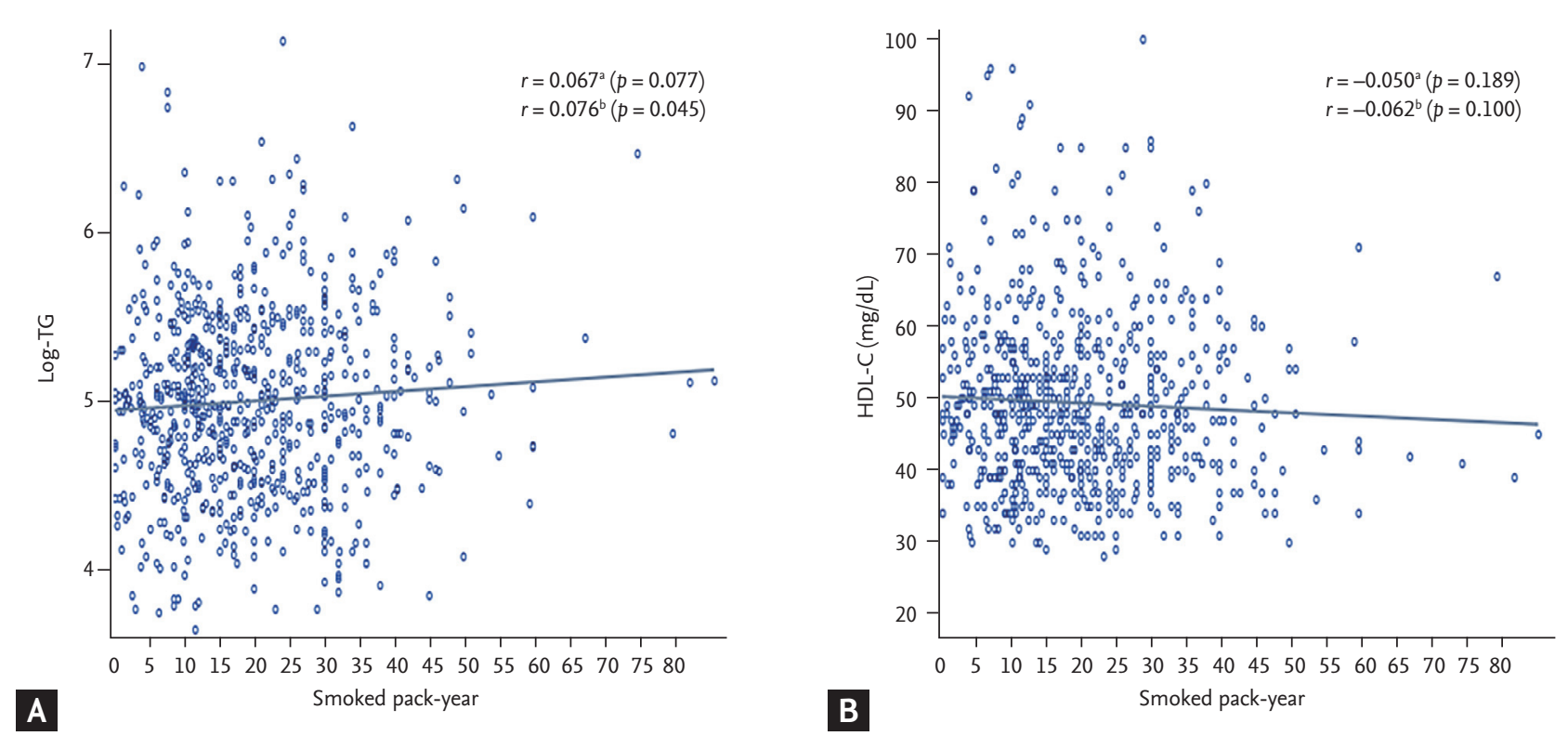

Figure 3. (A, B) Correlation between smoked pack-years and lipid levels among current smokers. TG, triglyceride; HDL-C, high-density lipoprotein cholesterol. 'Unadjusted Pearson's correlation coefficient. 'Adjusted Pearson's correlation coefficient, adjusted for age, body mass index, systolic blood pressure, fasting glucose, physical activity, and intake of energy, carbohydrate, and fat.

serum TG levels, and current smokers who had smoked more than 20 pack-years had much higher TG levels than individuals who had not. When we further divided smokers into light and heavy smoking groups using 20 pack-years as the cut-off, both current light and heavy smokers were found to have higher TG levels as compared to that of never-smokers, with a greater difference between current heavy smokers and never-smokers. Similarly, in other analyses, TG level was remarkably higher in current heavy smokers as compared to corresponding levels in other groups. Past heavy smokers were found to have a higher TG level in some analyses, as compared to never-smokers. For other lipid components, a lower HDL level was associated with current heavy smoking. Except for current heavy smokers, there was no significant difference in HDL-C level among other smoker groups. Meanwhile, for current smokers, a higher smoking amount was much worse than a lower amount. Our finding that male smokers had higher TG levels as compared to male non-smokers is consistent with the results of a number of previous studies [25-34]. Our results cohere with those reported in some previous studies that smokers with higher smoking amounts had higher TG levels as compared to individuals with relatively lower smoking amounts [28,33,34]. However, in several areas, our results were different from those reported previous; this applies to our finding that smoking had little impact on TG levels in men or that there was no significant difference in triglyceride levels between male smoking 'quitters' and smoking 'continuers' [35]. The lower level of HDL in smokers found in the present study was similar to the results of previous studies $[8-10,36,37]$. A recent review demonstrated that LDL-C is the least affected lipid component in smokers [38]; likewise, in the present study, we did not observe any significant association between cigarette smoking and LDL-C level.

In the present study, TG levels were found to be higher in current smokers, particularly in current heavy smokers. However, as revealed by baseline characteristics, the rate of high-risk drinkers among current smokers was $48.2 \%$, which is much higher than that among past smokers (29.9\%) and non-smokers (19.4\%); therefore, alcohol drinking status and smoking status are strongly related to each other. Although alcohol drinking level was adjusted in regression analysis, there is a possibility that "heavy alcohol drinkers," not "heavy smokers," have higher TG levels [39,40]. To test this possibility, we 
stratified the participants into three groups according to their current drinking status (non-drinkers, moderate drinkers, and high-risk drinkers) and re-analyzed the association between smoking status and TG levels. The results are reported in Supplementary Table 1. Most participants $(60.8 \%, \mathrm{n}=1,175)$ were moderate drinkers, and only $5.0 \%(n=97)$ were non-drinkers. In both moderate drinkers and high-risk drinkers, current light smoking and current heavy smoking were significantly associated with higher log-TG levels, which is similar with the result in the total sample $(n=1,932)$. However, this pattern was not observed in non-drinkers, which might be due to the small number of current smokers among highrisk drinkers.

Several previous studies revealed gender differences in how smoking affects lipid levels $[26,41,42]$; but the results of these studies were not conclusive. In order to investigate whether the association between cigarette smoking and serum lipid levels differs between two genders in our study population, we conducted additional analysis with a total of 3,747 women. Similarly to our results on male smokers, we found no significant association of smoking with TC and LDL-C in women. However, unlike the results on male smokers, female past light smokers had significantly higher HDL-C than female never-smokers, and there was a significantly strong interaction between gender and smoking status $(p=0.0282)$. Female current light smokers also had a significantly higher TG level than female never-smokers. However, unlike in male smokers, TG level of female past heavy smokers and current heavy smokers was not significantly different from that of never-smokers (Supplementary Table 2).

In the present study, information on nutritional intake was included as the covariate in the analysis of the association between smoking and lipid levels. Several previous studies reported that higher carbohydrate intake generally leads to lower HDL-C and higher TG [43-45]. In this study, a higher carbohydrate intake was associated with a lower HDL-C level, but TG was significantly lower in individuals with a higher carbohydrate intake, which is inconsistent with the results of previous studies. According to some studies, not only the quantity of carbohydrate consumed, but also the source and type of carbohydrate consumed are also (albeit differently) associated with cardiovascular risk factors $[46,47]$.
Of note, many studies on the relationship between carbohydrate intake and blood lipid levels have been conducted in Western countries. Considering that Asian and Western populations have very different aspects of carbohydrate intake [48], our results may differ from those of previous studies when it comes to the relationship between carbohydrate intake and TG level. Further research on dietary intake and lipid levels among the Korean population is needed.

Although the mechanism of how smoking affects serum lipid levels is not fully clear yet, several possible explanations can be proposed. In one model, rising catecholamine levels may cause a surge in circulating free fatty acids that stimulates hepatic TG synthesis and changes lipid metabolism [49,50]. Another model is that smoking evokes reduced activity of lecithin-cholesterol acyltransferase, the enzyme in charge of esterifying free cholesterol, which results in altered lipid metabolism [37,51]. The third possibility of higher TG level in smokers is that TG metabolism is slower in smokers, as lipoprotein lipase activity, which affects TG levels, is decreased in smokers [52].

The present study has several strengths compared to previous studies. First, our sample included apparently healthy subjects free of major CVD. Therefore, we investigated the impact of smoking on blood lipid profiles before progression to clinically apparent cardiovascular pathogenesis. Second, we identified an independent association between smoking and blood lipid levels by excluding individuals taking any lipid-control medications or receiving other treatments, as well as by additionally adjusting for variables that may influence lipid levels. Third, few previous studies that evaluated the effect of smoking on blood lipid profiles have accounted for nutrient intake and physical activity. Since our analysis considered the effect of these variables on lipid levels, we were able to more directly evaluate the independent association between cigarette smoking and blood lipid levels. Finally, our study adequately reflects the smoking behavior of the general population in Korea. According to the 2017 Korean National Health and Nutrition Survey data, among the men in the same age (30 to 64 years old), current smoking rate was $37.25 \%$, and past smoking rate was $33.43 \%$, with a lifetime smoking experience rate of $70.68 \%$. The lifetime smoking experience rate of our study population was $76.80 \%$, which is 
similar to that of the general population. Therefore, it is expected that the results of this study can be extended to general population.

Our study has several limitations. First, due to the cross-sectional study design, we could not investigate the longitudinal effect of cigarette smoking on blood lipid levels. Future longitudinal research could confirm that increased serum TG levels and decreased HDL level result from smoking habits and establish a cause-andeffect relationship. Second, considering that information on smoking was obtained by in-person interviews, inaccuracy may be present in the data from measurement errors and recall bias [53]. However, current smoking status is not likely to be an object of recall bias. Additionally, in order to determine the dose of smoking in pack-years, we examined the reliability of two types of information: age at smoking initiation and amount of smoking per day. A part of our study population had received health examinations in years 1999, 2005, or 2010 to 2011 in a separate study. Smoking data obtained in 1999, 2005, or 2010 to 2011 examinations were matched with smoking data from the CMERC cohort. A total of 135 pairs were derived from these data, and Spearman's correlation analyses were conducted. The correlation coefficients for the age at smoking initiation and for the amount of smoking per day were $0.49983(p<0.001)$ and $0.50121(p<0.001)$, respectively. Third, there could have been unknown or unexpected confounding variables in lifestyles between smokers and never-smokers. However, major causes of dyslipidemia, including obesity, alcohol intake, diabetes, and other lifestyle factors, were considered in the present study. Fourth, we could not measure the impact of passive smoking. According to previous studies, passive smoking is associated with increased risk of CVD [54,55]; however, its effect on CVD is much smaller than that of active smoking, according to a meta-analysis study [55].

In Korea, the proportion of men aged over 30 years old and having hypertriglyceridemia is more than twice higher than that of women (24.8\% vs. $11.0 \%)$ [11]. These gender differences might be attributable to the differences in the practice of health behaviors between two genders, such as alcohol drinking pattern and cigarette smoking. As hypertriglyceridemia is an independent risk factor for CVD including coronary heart disease
[56,57], the management of TG is crucial. In this regard, the results of the present study are meaningful because we demonstrated that cigarette smoking alone increases TG level, even after ruling out the effects of commonly-known risk factors (obesity, blood pressure, glucose, nutritional intake, alcohol drinking, and exercise) on TG level. Furthermore, the proportion of Korean men over 30 with hypo-HDL cholesterolemia amounts to 27.2\% [11]. Elevating HDL-C is helpful for the prevention of CVD, as it is known to be a "protective particle" that improves endothelial function and limits atherosclerosis progression $[10,50]$. In the present study, current heavy smoking was shown to be associated with a lower HDL-C level, which suggests the possibility that cigarette smoking might be a risk factor for lowering HDL-C.

In conclusion, in the present study, we found that current cigarette smoking is associated with high TG levels in apparently healthy Korean males. Additionally, we found that people with a higher number of cigarettes smoked in pack-years had higher serum TG levels. For current smokers with over 20 pack-years, not only higher TG, but also lower HDL-C were observed. The lipid levels of past smokers were not considerably different from those of never-smokers. Further research is needed to clarify the effect of cigarette smoking on blood lipid profiles and to discover the mechanism by which smoking alters lipid metabolism.

\section{KEY MESSAGE}

1. In apparently healthy Korean male population, current cigarette smoking was found to be associated with higher serum triglyceride (TG) levels, and heavy smokers had higher TG levels than light smokers.

2. Current smokers with over 20 pack-years had not only higher log-triglycerides levels, but also lower high-density lipoprotein levels.

3. The lipid levels of past smokers (particularly past smokers with fewer than 20 pack-years) were almost identical to those of never-smokers. 


\section{Conflict of interest}

No potential conflict of interest relevant to this article was reported.

\section{Acknowledgments}

This work was supported by a grant of the Korea Health Technology R\&D Project through the Korea Health Industry Development Institute (KHIDI), funded by the Ministry of Health and Welfare, Republic of Korea (grant no. HI13Co715).

\section{REFERENCES}

1. Grundy SM, Becker D, Clark LT, et al. Detection, Evaluation, and Treatment of High Blood Cholesterol in Adults (Adult Treatment Panel III). Bethesda (MD): National Institutes of Health, 2002:1-28.

2. McGill HC Jr, McMahan CA, Malcom GT, Oalmann MC, Strong JP. Effects of serum lipoproteins and smoking on atherosclerosis in young men and women. The PDAY Research Group. Pathobiological Determinants of Atherosclerosis in Youth. Arterioscler Thromb Vasc Biol 1997;17:95-106.

3. Castelli WP, Garrison RJ, Wilson PW, Abbott RD, Kalousdian S, Kannel WB. Incidence of coronary heart disease and lipoprotein cholesterol levels. The Framingham Study. JAMA 1986;256:2835-2838.

4. Manninen V, Tenkanen L, Koskinen P, et al. Joint effects of serum triglyceride and LDL cholesterol and HDL cholesterol concentrations on coronary heart disease risk in the Helsinki Heart Study. Implications for treatment. Circulation 1992;85:37-45.

5. Kannel WB. Some lessons in cardiovascular epidemiology from Framingham. Am J Cardiol 1976;37:269-282.

6. Hammond EC, Horn D. Landmark article March 15, 1958: Smoking an d death rates: report on forty-four months of follow-up of 187,783 men. By E. Cuyler Hammond and Daniel Horn. JAMA 1984;251:2840-2853.

7. Pasupathi P, Rao YY, Farook J, Saravanan G, Bakthavathsalam G. Effect of cigarette smoking on lipids and oxidative stress biomarkers in patients with acute myocardial infarction. Res J Med Med Sci 2009;4:151-159.

8. Garrison RJ, Kannel WB, Feinleib M, Castelli WP, McNamara PM, Padgett SJ. Cigarette smoking and HDL cholesterol: the Framingham offspring study. Atheroscle- rosis 1978;30:17-25.

9. Freeman DJ, Griffin BA, Murray E, et al. Smoking and plasma lipoproteins in man: effects on low density lipoprotein cholesterol levels and high density lipoprotein subfraction distribution. Eur J Clin Invest 1993;23:630640.

10. Ludicke F, Magnette J, Baker G, Weitkunat R. A Japanese cross-sectional multicentre study of biomarkers associated with cardiovascular disease in smokers and non-smokers. Biomarkers 2015;20:411-421.

11. The Korean Society of Lipid and Atherosclerosis. Dyslipidemia Fact Sheets in Korea, 2018. Seoul (KR): The Korean Society of Lipid and Atherosclerosis, 2018.

12. Organization for Economic Cooperation and Development. Health at a Glance 2017 [Internet]. Paris (FR): OECD, c2019 [cited 2019 Nov 14]. Available from: https:// doi.org/10.1787/health_glance-2017-en.

13. Shim JS, Song BM, Lee JH, et al. Cardiovascular and Metabolic Diseases Etiology Research Center (CMERC) cohort: study protocol and results of the first 3 years of enrollment. Epidemiol Health 2017;39:e2017016.

14. Office of the Surgeon General; Centers for Disease Control and Prevention (US); National Center for Chronic Disease Prevention and Health Promotion (US) Office on Smoking and Health. How Tobacco Smoke Causes Disease: The Biology and Behavioral Basis for Smoking-Attributable Disease: A Report Of The Surgeon General. Rockville (MD): Centers for Disease Control \& Prevention, 2010.

15. Hughes JR, Peters EN, Naud S. Relapse to smoking after 1 year of abstinence: a meta-analysis. Addict Behav 2008;33:1516-1520.

16. Pastorino U, Bellomi M, Landoni C, et al. Early lung-cancer detection with spiral CT and positron emission tomography in heavy smokers: 2-year results. Lancet 2003;362:593-597.

17. Hassan MM, Phan A, Li D, Dagohoy CG, Leary C, Yao JC. Risk factors associated with neuroendocrine tumors: a U.S.-based case-control study. Int J Cancer 2008;123:867873.

18. Anandha Lakshmi S, Lakshmanan A, Ganesh Kumar P, Saravanan A. Effect of intensity of cigarette smoking on haematological and lipid parameters. J Clin Diagn Res 2014;8:BC11- BC13.

19. Neumann T, Rasmussen M, Heitmann BL, Tonnesen H. Gold standard program for heavy smokers in a real-life 
setting. Int J Environ Res Public Health 2013;10:4186-4199.

20. Kweon S, Kim Y, Jang MJ, et al. Data resource profile: the Korea National Health and Nutrition Examination Survey (KNHANES). Int J Epidemiol 2014;43:69-77.

21. IPAQ Research Committee. Guidelines for data processing and analysis of the International Physical Activity Questionnaire (IPAQ)-short and long forms [Internet]. IPAQ, 2019 [cited 2019 Nov 14]. Available from: http://www. ipaq.ki.se.

22. Oh JY, Yang YJ, Kim BS, Kang JH. Validity and reliability of Korean version of International Physical Activity Questionnaire (IPAQ) short form. J Korean Acad Fam Med 2007;28:532-541.

23. Friedewald WT, Levy RI, Fredrickson DS. Estimation of the concentration of low-density lipoprotein cholesterol in plasma, without use of the preparative ultracentrifuge. Clin Chem 1972;18:499-502.

24. Lenth RV. Least-squares means: the R package lsmeans. J Stat Softw 2016;69:1-33.

25. Billimoria JD, Pozner H, Metselaar B, Best FW, James DC. Effect of cigarette smoking on lipids, lipoproteins, blood coagulation, fibrinolysis and cellular components of human blood. Atherosclerosis 1975;21:61-76.

26. Lee MH, Ahn SV, Hur NW, Choi DP, Kim HC, Suh I. Gender differences in the association between smoking and dyslipidemia: 2005 Korean National Health and Nutrition Examination Survey. Clin Chim Acta 2011;412:1600-1605.

27. Sharma SB, Dwivedi S, Prabhu KM, Singh G, Kumar N, Lal MK. Coronary risk variables in young asymptomatic smokers. Indian J Med Res 2005;122:205-210.

28. Brischetto CS, Connor WE, Connor SL, Matarazzo JD. Plasma lipid and lipoprotein profiles of cigarette smokers from randomly selected families: enhancement of hyperlipidemia and depression of high-density lipoprotein. Am J Cardiol 1983;52:675-680.

29. Taylor KG, Carter TJ, Valente AJ, Wright AD, Smith JH, Matthews KA. Sex differences in the relationships between obesity, alcohol consumption and cigarette smoking and serum lipid and apolipoprotein concentrations in a normal population. Atherosclerosis 1981;38:11-18.

30. Axelsen M, Eliasson B, Joheim E, Lenner RA, Taskinen MR, Smith U. Lipid intolerance in smokers. J Intern Med 1995;237:449-455.

31. Hautanen A, Adlercreutz H. Hyperinsulinaemia, dyslipidaemia and exaggerated adrenal androgen response to adrenocorticotropin in male smokers. Diabetologia
1993;36:1275-1281.

32. Kuzuya M, Ando F, Iguchi A, Shimokata H. Effect of smoking habit on age-related changes in serum lipids: a cross-sectional and longitudinal analysis in a large Japanese cohort. Atherosclerosis 2006;185:183-190.

33. Meenakshisundaram R, Rajendiran C, Thirumalaikolundusubramanian P. Lipid and lipoprotein profiles among middle aged male smokers: a study from southern India. Tob Induc Dis 2010;8:11.

34. Kume A, Kume T, Masuda K, Shibuya F, Yamazaki H. Dose-dependent effects of cigarette smoke on blood biomarkers in healthy Japanese volunteers: observations from smoking and non-smoking. J Health Sci 2009;55:259-264.

35. Yoon C, Goh E, Park SM, Cho B. Effects of smoking cessation and weight gain on cardiovascular disease risk factors in Asian male population. Atherosclerosis 2010;208:275-279.

36. Moriguchi EH, Fusegawa Y, Tamachi H, Goto Y. Effects of smoking on HDL subfractions in myocardial infarction patients: effects on lecithin-cholesterol acyltransferase and hepatic lipase. Clin Chim Acta 1991;195:139-143.

37. McCall MR, van den Berg JJ, Kuypers FA, et al. Modification of LCAT activity and HDL structure. New links between cigarette smoke and coronary heart disease risk. Arterioscler Thromb 1994;14:248-253.

38. Craig WY, Palomaki GE, Haddow JE. Cigarette smoking and serum lipid and lipoprotein concentrations: an analysis of published data. BMJ 1989;298:784-788.

39. Contaldo F, D'Arrigo E, Carandente V, et al. Short-term effects of moderate alcohol consumption on lipid metabolism and energy balance in normal men. Metabolism 1989;38:166-171.

40. Crouse JR, Grundy SM. Effects of alcohol on plasma lipoproteins and cholesterol and triglyceride metabolism in man. J Lipid Res 1984;25:486-496.

41. Vriz O, Nesbitt S, Krause L, Majahalme S, Lu H, Julius S. Smoking is associated with higher cardiovascular risk in young women than in men: the Tecumseh Blood Pressure Study. J Hypertens 1997;15:127-134.

42. Namekata T, Moore DE, Suzuki K, et al. Biological and lifestyle factors, and lipid and lipoprotein levels among Japanese Americans in Seattle and Japanese men in Japan. Int J Epidemiol 1997;26:1203-1213.

43. Ma Y, Li Y, Chiriboga DE, et al. Association between carbohydrate intake and serum lipids. J Am Coll Nutr 
2006;25:155-163.

44. Foster GD, Wyatt HR, Hill JO, et al. A randomized trial of a low-carbohydrate diet for obesity. N Engl J Med 2003;348:2082-2090.

45. Merchant AT, Anand SS, Kelemen LE, et al. Carbohydrate intake and HDL in a multiethnic population. Am J Clin Nutr 2007;85:225-230.

46. Song S, Lee JE, Song WO, Paik HY, Song Y. Carbohydrate intake and refined-grain consumption are associated with metabolic syndrome in the Korean adult population. J Acad Nutr Diet 2014;114:54-62.

47. Jung SJ, Kim MG, Park TS, Kim YG, Song WO, Chae SW. Rice-based Korean meals (bibimbap and kimbap) have lower glycemic responses and postprandial-triglyceride effects than energy-matched Western meals. J Ethn Foods 2015;2:154-161.

48. Choi H, Song S, Kim J, et al. High carbohydrate intake was inversely associated with high-density lipoprotein cholesterol among Korean adults. Nutr Res 2012;32:100106.

49. Freedman DS, Srinivasan SR, Shear CL, et al. Cigarette smoking initiation and longitudinal changes in serum lipids and lipoproteins in early adulthood: the Bogalusa Heart Study. Am J Epidemiol 1986;124:207-219.

50. Chelland Campbell S, Moffatt RJ, Stamford BA. Smoking and smoking cessation: the relationship between cardiovascular disease and lipoprotein metabolism: a review. Atherosclerosis 2008;201:225-235.
51. Gepner AD, Piper ME, Johnson HM, Fiore MC, Baker TB, Stein JH. Effects of smoking and smoking cessation on lipids and lipoproteins: outcomes from a randomized clinical trial. Am Heart J 2011;161:145-151.

52. Freeman DJ, Caslake MJ, Griffin BA, et al. The effect of smoking on post-heparin lipoprotein and hepatic lipase, cholesteryl ester transfer protein and lecithin:cholesterol acyl transferase activities in human plasma. Eur J Clin Invest 1998;28:584-591.

53. Tverdal A, Thelle D, Stensvold I, Leren P, Bjartveit K. Mortality in relation to smoking history: 13 years' follow-up of 68,00o Norwegian men and women 35-49 years. J Clin Epidemiol 1993;46:475-487.

54. Celermajer DS, Adams MR, Clarkson P, et al. Passive smoking and impaired endothelium-dependent arterial dilatation in healthy young adults. $\mathrm{N}$ Engl J Med 1996;334:150-154.

55. He J, Vupputuri S, Allen K, Prerost MR, Hughes J, Whelton PK. Passive smoking and the risk of coronary heart disease: a meta-analysis of epidemiologic studies. N Engl J Med 1999;340:920-926.

56. Austin MA, Hokanson JE, Edwards KL. Hypertriglyceridemia as a cardiovascular risk factor. Am J Cardiol 1998;81:7B-12B.

57. Sarwar N, Danesh J, Eiriksdottir G, et al. Triglycerides and the risk of coronary heart disease: 10,158 incident cases among 262,525 participants in 29 Western prospective studies. Circulation 2007;115:450-458. 


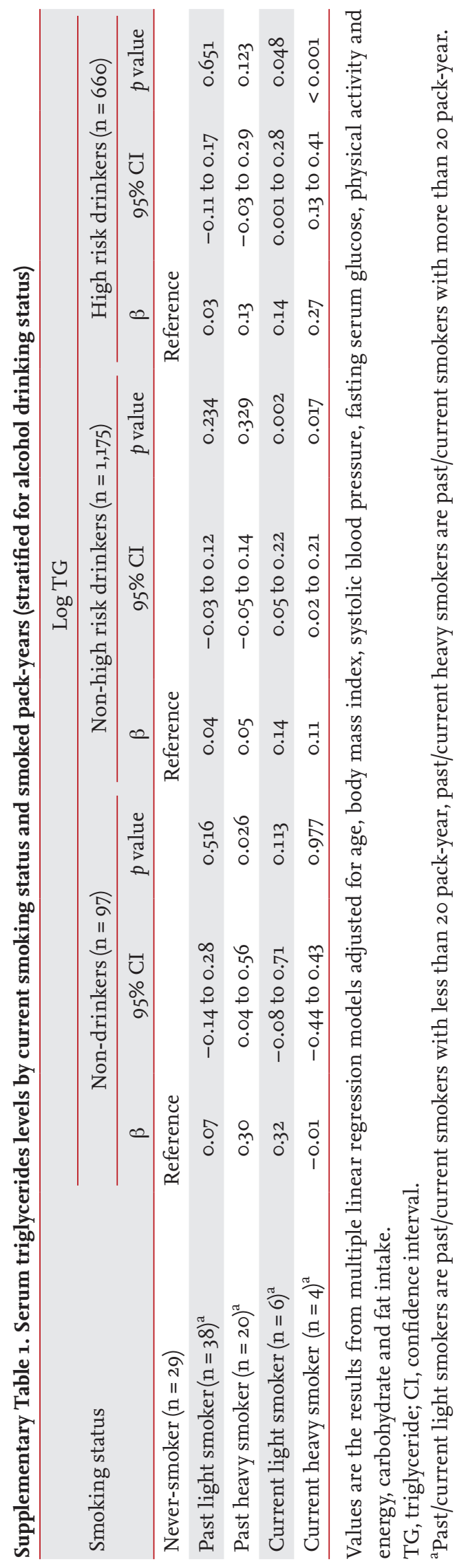




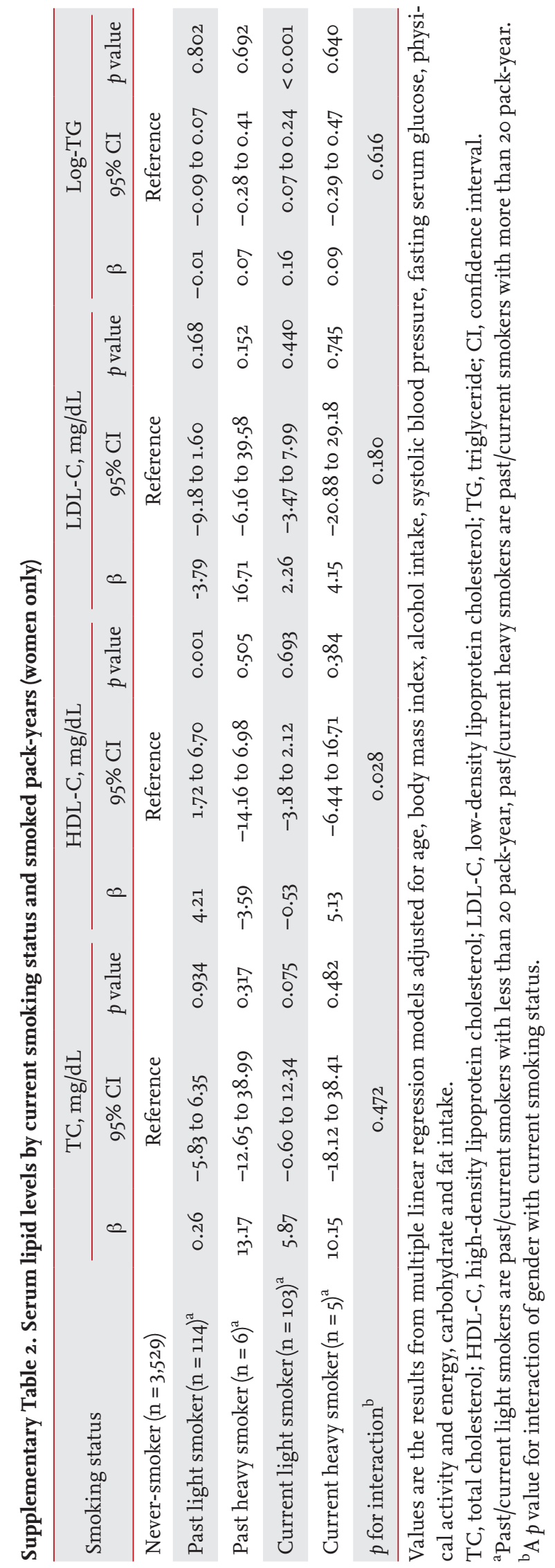

